\title{
The development and psychometric properties of the Arabic version of the child oral health impact profile-short form (COHIP- SF 19)
}

\author{
A. A. Arheiam ${ }^{1,3^{*}}$, S. R. Baker ${ }^{2}$, L. Ballo ${ }^{3}$, I. Elareibi ${ }^{3}$, S. Fakron ${ }^{3}$ and R. V. Harris ${ }^{1}$
}

\begin{abstract}
Background: This study aims to cross-culturally adapt the original English-language COHIP-SF 19 to Arabic culture and to test its psychometric properties in a community sample.

Methods: The Arabic COHIP-SF 19 was developed and its psychometric properties were examined in a populationbased sample of 876 schoolchildren who were aged 12 years of age, in Benghazi, Libya. The Arabic COHIP-SF 19 was tested for its internal consistency, reproducibility, construct validity, factorial validity and floor as well as ceiling effects. A Mann-Whitney U test was used to compare the mean scores of COHIP-SF 19 by participants' caries status and self-reported oral health rating, satisfaction and treatment need.

Results: The Arabic COHIP-SF 19 was successfully and smoothly developed. It showed an acceptable level of equivalence to the original version. Overall, the internal consistency and reproducibility were acceptable to excellent, with a Cronbach's alpha of 0.84 and an intra-class correlation coefficient (ICC) of 0.76. All hypotheses predefined to test construct validity were confirmed. That is, children who had active dental caries, and who rated their oral health as poor, were not satisfied with their oral health or indicated the need of treatment had lower COHIP-SF 19 scores $(P<0.05)$. Floor or ceiling effects were not observed. The exploratory Factorial analysis suggested a 4-component solution and deletion of one item.
\end{abstract}

Conclusion: The Arabic COHIP-SF 19 was successfully developed. The measure demonstrated satisfactory reliability and validity to estimate OHRQOL in a representative sample of 12-year-old schoolchildren.

Keywords: Quality of life, Oral health, Reliability, Validity, Arabic, Children

\section{Background}

Oral health-related quality of life (OHRQoL) is a multidimensional, patient-centered subjective measure of functional and psycho-social impacts of oral health [1]. Recently, a new definition of oral health has been adopted by the Dental Federation General Assembly has acknowledged psychosocial function as a core element of oral health which a multifaceted construct [2]. This movement comes as no surprise since the

\footnotetext{
* Correspondence: Arheiam@gmail.com

'Department of Health Services Research University of Liverpool, Liverpool, UK

${ }^{3}$ Department of Community and Preventive Dentistry, Faculty of Dentistry, University of Benghazi, Benghazi, Libya

Full list of author information is available at the end of the article
}

psychosocial impacts of oral health have been the center of attention in the dental literature for some time now, in recognition of a paradigm shift in defining oral health needs and outcomes from a narrow biomedical to a wider biopsychosocial approach [3]. Many OHRQoL measures have been developed and used for oral health assessment, to supplement conventional clinical indicators $[3,4]$. Amongst the various important theoretical, political and practical applications of OHRQoL measures [5], their use in epidemiological surveys has become increasingly popular $[3,6,7]$. The useof OHRQoL measures has huge implications for oral health services planning, evaluation and allocation of resources and 
decision making [8-11]; leading in due course to more efficient service planning $[3,4]$.

Dental caries is a major public health problem in many developing and developed countries, with significant impacts on quality of life, particularly among children $[12,13]$. Dental caries can cause severe tooth pain [14, 15], sepsis and tooth extraction [16], and consequently significant impact on school attendance [17], and self-esteem of children [18]. Although many measures have been developed to assess OHRQoL among school age children $[19,20]$, the Child Oral Health Impact Profile (COHIP) stands out for being both suitable for children between 8 and 15 years of age, while also evaluating both positive and negative attributes of quality of life [21]. What is more, recently, a shorter version of COHIP (COHIP-SF19) has been developed using a confirmatory factor analysis [22]. Such short forms are appropriate for large surveys since they are less time consuming, easy to use and interpret and consequently more cost-effective [23].

However, since the initial development by Broder et al. in 2012, there has been very little published research on the cross-cultural adaptation and validation of COHIPSF 19 . To the authors' knowledge, only one study has addressed this issue which was conducted in China [24]. Every time an OHRQoL measure is used in a different context or cultural group, it needs to be cross-culturally adapted and tested for its psychometric properties [25-27]. This procedure aims to ensure the suitability of the OHRQoL measure to the new context as well as its equivalence to the original measure. Herdman et al. (1998) [26] proposed a framework of six aspects of equivalence, defined in Table 1 (semantic, conceptual, item, operational, measurement and functional), to be considered when cross-culturally adapting quality of life questionnaires.

Given that there are few child OHRQoL measures translated to Arabic (CPQ11-14 \&C-OIPD) [19], and that no previous attempts have been made to develop an Arabic version of COHIP-SF 19; this study was conducted to cross-culturally adapt the original English- language COHIP-SF 19 to Arabic culture and to test its psychometric properties in population-based sample of 12-year-old schoolchildren in Libya.

\section{Methods and results}

Ethical clearance and permissions for the study were obtained from ethics committee at the University of Liverpool and faculty of Dentistry at the University of Benghazi prior to data collection. Written informed consents were obtained from the parents/guardians. In this paper, the methods and results section are combined in one section to reflect the sequence of procedures employed in the cross-cultural adaptation and psychometric testing of Arabic COHIP-SF19, according to the guidelines proposed by Beaton et al. (2000) [25].

\section{Stage 1: Translation of the original COHIP-SF19}

The original English-Language COHIP-SF19 (OV) was translated to the Arabic language using a rigorous forward-backward translation process. The OV was first translated into the Arabic language by two bilingual native Arabic speakers (an English language teacher and a dentist who lived for many in years in the UK). The translators worked independently and were preinformed about the aim of the questionnaire and its target group. They were also requested to identify any 'difficult to translate' words. The two Arabic translations (T1 \&T2) were then discussed with the research team to be consolidated in one Arabic version (T12). This process was then repeated the other way around. The Arabic version (T12) was translated into the English language by two native English speakers who speak Arabic fluently. Two independent translations (BT1 \& BT2) were created, which were then discussed with the investigators to generate one English version (BT12). A committee of experts reviewed the translations and assessed its semantic equivalence to the OV [26], to approve a pre-final version of the Arabic COHIP-SF19. The committee of experts included a languages expert, the translators, two dentists and a dental researcher in the area of quality of life and two native English speakers [25].

Table 1 Definition of aspects of equivalence according to Hardman (1998) [26], extracted from Saub et al. (2007) [35]

\begin{tabular}{ll}
\hline Equivalence & Definition \\
\hline Conceptual & $\begin{array}{l}\text { Ways in which different populations conceptualize health and quality of life (QoL) and the values they place } \\
\text { on different domains of health and QoL. } \\
\text { Item }\end{array}$ \\
& $\begin{array}{l}\text { Concerns the way in which domains are sampled. Item equivalence exists when items estimate the same parameters } \\
\text { on the latent trait being measured and when they are equally relevant and acceptable in both cultures. } \\
\text { Operational }\end{array}$ \\
& $\begin{array}{l}\text { Concerned with the transfer of meaning across languages. } \\
\text { and measurement method (response format). }\end{array}$ \\
Measurement & Ensuring that different language versions of the same instrument achieve acceptable levels in terms \\
Functional & The extent to which an instrument does what it is supposed to do equally well in two or more cultures.
\end{tabular}


None of the questionnaire items were found challenging for the translators or required a modification. The committee was satisfied with the Arabic version produced and no major or meaning related modifications were suggested. The pre-final version of Arabic COHIPSF 19 was approved by the committee of experts. It comprised 19 items distributed over 3 conceptual subscales as following: Oral health (5 items), Functional well-being (4 items), and Socio-emotional well-being (10 items). A five-point Likert scale ('never' $=0$, 'almost never' $=1$, 'sometimes' $=2$, 'fairly often' $=3$, and 'almost all of the time' $=4$.) was used to collect responses for all items. The question: 'How often have you experienced oral impacts during the past 3 months?' was posed at the outset of the questionnaire. After reversing the scoring of the 17 negatively-worded items, the total score ranged from 0 to 76 , with the higher score indicating better quality of life.

\section{Stage 2: Testing of pre-final Arabic COHIP-SF 19}

The pre-final Arabic COHIP-SF19 was tested for its conceptual, item and operational equivalence (Table 1). The questionnaire was piloted at the department of paediatric dentistry at the Faculty of Dentistry at the University of Benghazi. A separate group of 35 children who were not participants in the stage 3 study were asked to complete the questionnaire. Also, one-to-one interviews were conducted, in the presence of their parents, to explore children's views regarding each item in terms of meaning, clarity of wording, relevance to oral health and its conceptual subscale and the response options. Based on the feedback received from the participants, a final Arabic COHIP-SF 19 was produced.

All the items were considered relevant and clearly understood. The domains were identical to the OV. No changes in the response options or the questionnaire format or mode of administration were suggested. The final Arabic COHIP-SF19 was pre-tested and produced.

Stage 3: Psychometric properties of Arabic COHIP-SF 19

After the cross-cultural adaptation, it is highly recommended that the new version is tested for its measurement properties among its target population [25]. To do so, a cross-sectional study design was used to examine the psychometric properties of the Arabic COHIP-SF 19 in a population-based sample of 12-year-old Libyan schoolchildren. This study was part of a survey investigating oral health status and treatment needs in conflictaffected Libya and to compare these with pre-conflict data. Therefore, the survey aimed to collect data from a comparable sample size which was identified to be at least 800 . Only procedures related to testing the psychometric properties of Arabic COHIP-SF 19 are reported here.

\section{Study sample}

The participants were 12-year-old school children registered in the sixth grade for the academic year 2016/17 in Benghazi, Libya. The sampling frame was a total of 12,761 children, with almost equal male and female distribution, registered in 40 state-run schools distributed over 8 main districts. The participants were recruited by using a multi-stage clustering random sampling technique, using the schools as the clustering unit. At the first stage, a proportional sample of schools was randomly selected from each district. At the second stage, children were randomly selected from each school. The random selection of schools and participants was chosen by using computer system. A minimum sample size of 400 had previously been identified to be sufficient for studies assessing reliability and validity [28]. In the present study, a total of 950 participants were recruited to take part, from 16 schools.

\section{Questionnaire administration}

The children's schools were first approached to arrange for data collection. Informed consent was first sought from the parents which was sent to them through the school administration. Only participants with parental consent were included in the study. The Arabic COHIPSF 19 was administered on a separate day by trained research assistants in quiet rooms in their schools, after explaining the aim of the study. Verbal assent was obtained from the children and implied by them returning completed questionnaires and attending the dental examination. The Arabic COHIP-SF 19 was provided along with another questionnaire covering oral health behaviors and sociodemographic information. Trained research assistants were available on demand at the research sites to aid the participants in completing the questionnaire. All participants took a maximum of $10 \mathrm{~min}$ to complete the questionnaire. The Arabic COHIP-SF 19 was administered again after 3 weeks to a sub-sample of 100 participants, randomly selected from 4 schools. This step was undertaken to allow the assessment of the measure's reproducibility.

\section{Clinical examination}

Three dentists were trained and calibrated to carry out the clinical dental examinations. The training sessions were provided at the department of Community and Preventive Dentistry, University of Benghazi. Intraexaminer reliability and inter-examiner reliability were tested in a separate group of 12-year-old school children before commencing the data collection of the main study. Kappa coefficient ranged from 0.82 to 0.96 . After completing the questionnaires, dental examination was conducted for all participants in a separate room under daylight while the participant was seated on an ordinary 
chair. The children were assessed for their oral health status and treatment needs according to WHO diagnostic criteria and forms, using disposable diagnostic kits. Dental caries experience was assessed at dentine level (Cavitation) using the DMFT and DMFS indices [29].

\section{Data analysis}

Of 950 children recruited for the study, 876 participants provided complete questionnaires usable for analysis. All data analyses were conducted using SPSS software (IBM, Version 24). Internal consistency was assessed by calculating Cronbach's alpha coefficient for the overall scale and for each subscale (Oral health, Functional wellbeing and Socio-emotional well-being). Cronbach's alpha values $\geq 0.6$ was considered as an acceptable level [30]. The intra-class correlation coefficients (ICC) were used to assess test-retest reliability. These were calculated for scores from the repeated administrations of the questionnaire. An ICC of 0.7 indicates an acceptable level of reproducibility [19].

Construct validity of Arabic COHIP-SF 19 was evaluated by examining measures of the discriminant and convergent validity [22]. These were examined against 4 predefined hypotheses [31], as following: lower COHIPSF 19 scores would be observed among those who 1) perceived their oral health as poor; 2) were not satisfied with their oral health; 3) indicated the need for dental treatment; 4) had active dental caries (had more than one decayed tooth vs caries-free). To test these hypotheses, the participants were asked to answer 3 general questions on whether they were satisfied with their oral health (Satisfied VS not-satisfied), whether they perceived any need for oral health treatment (Yes VS No) and how they rated their own oral health (good/excellent VS poor). All hypotheses were tested by employing Mann-Whitney $\mathrm{U}$ test at $p<0.05$.

An exploratory factor analysis (EFA) was conducted to test the factorial validity of items in the subscales defined in the original COHIP-SF19, using the varimax rotation and a strict cut-off of factor loading of $>0.50$ [32]. Item-impact values for the scale items were computed as the product of the mean score and percentage of participants generally had that impact ('sometimes' $=2$, 'fairly often' $=3$, and 'almost all of the time' $=4$ responses on the item) [33]. The purpose of the item impact phase was to measure the prevalence and importance of the scale items in the Arabic culture.

The questionnaire was also tested for the existence of ceiling or floor effects by calculating the frequencies of participants who achieved the lowest or highest possible score. If more than $15 \%$ of participants achieved the lowest or highest possible score, the Arabic COHIP-SF 19 was considered to have floor or ceiling effects respectively [31].

\section{Results of stage 3}

Distribution and comparison by gender of Arabic COHIP-SF 19 scores Table 2 shows the distribution of Arabic COHIP-SF19 scores and the subscales. The mean overall score was 61.13 (12.97) and ranged between 4 and 76. Scores for the overall scale and Oral health and Functional wellbeing subscales were significantly $(P<0.05)$ lower among female participants than that in males. The score of Social-emotional wellbeing subscale was also higher in males, although this was not statistically significant (Fig. 1).

Internal consistency and test-retest reliability The overall Cronbach's alpha of Arabic COHIP-SF 19 was 0.85. For the subscales, Cronbach's alpha was $0.65,0.69$ and 0.84 for the Oral health, Functional well-being and Socio-emotional well-being scales respectively. Generally, Cronbach's alpha did not improve when any of the items were removed from the scale. The corrected itemtotal correlations were positive, ranging from 0.19 to 0.72 . ICC for the overall scale and the subscales ranged between 0.70 and 0.76 (Table 2).

Construct validity Table 3 presents comparisons of mean scores of Arabic-COHIP-SF 19 and its subscales by participants, caries status and oral health satisfaction, rating and perceived treatment need. The mean score of the overall scale and the subscales of Arabic COHIP-SF 19 were significantly higher among those who rated their oral

Table 2 Summary reliability measures and descriptive statistics for Arabic-COHIP-SF 19 and subscale scores $(n=876)$

\begin{tabular}{|c|c|c|c|c|}
\hline \multirow[t]{2}{*}{ Descriptive statistics } & \multicolumn{4}{|c|}{ Overall scale, subscales (No. of Items) } \\
\hline & Overall scale & Oral health well-being & Functional well-being & Socio-emotional well-being \\
\hline$\overline{\text { Mean }(S D)^{b}}$ & $61.13(12.97)$ & $14.82(4.58)$ & $14.31(3.01)$ & $32.01(8.45)$ \\
\hline Range & $4-76$ & $0-20$ & $0-16$ & $0-40$ \\
\hline Proportion of lowest possible score ${ }^{a}$ & $0(0)$ & $10(1.1)$ & $8(0.9)$ & $6(0.7)$ \\
\hline Proportion of highest possible score ${ }^{a}$ & $59(6.7)$ & $185(21.1)$ & $548(62.6)$ & $244(27.9)$ \\
\hline Cronbach's alpha & 0.85 & 0.64 & 0.69 & 0.83 \\
\hline Alpha if an item is deleted & $0.83-0.86$ & $0.57-0.61$ & $0.59-0.67$ & $0.80-0.85$ \\
\hline
\end{tabular}

${ }^{a}$ Count (\%), ${ }^{b}$ higher score indicates better quality of life 


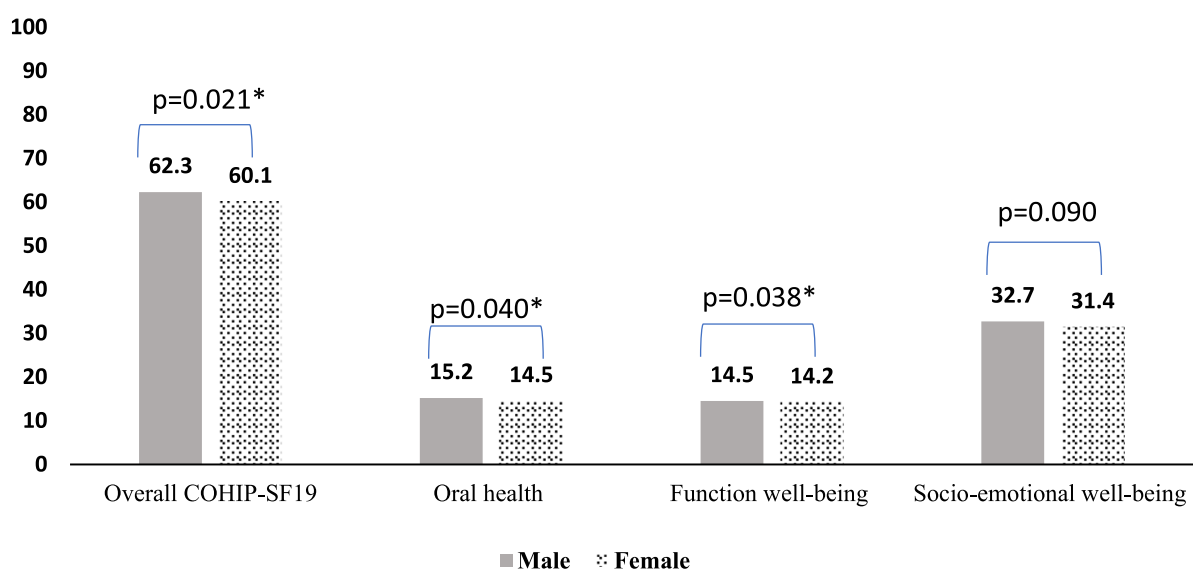

Fig. 1 Comparison of overall COHIP-SF19 and its subscales by participants' gender. Man-Whitney $\mathrm{U}$ test was used to compare the subgroups, * $P \leq 0.05$

health as 'good/excellent' than among those who perceived oral health as 'poor/very poor' $(p>0.001)$. The mean scores of Arabic COHIP-SF 19 and its subscales were significantly lower $(p>0.001)$ among children who did not feel satisfied with their oral health and who indicated the need of dental treatment (Table 3). Comparisons of mean scores across caries activity subgroups showed higher scores among caries-free children, which was statistically significant $(p>0.05)$ for the overall scale as well as Oral health and Functional well-being subscales but not for the Socio-emotional well-being subscale (Table 4).
Ceiling \& floor effects None of the participants achieved the lowest possible score (0) for the overall scale, whereas $6.7 \%$ of the participants achieved the highest possible score. For the subscales, the highest possible score was most commonly achieved in the Functional well-being subscale (67.5\%). On the other hand, the numbers of those who achieved the lowest possible score were generally low in all subscales, and ranged between 6 and 10 participants (Table 2).

Table 4 presents the EFA and item-impact analysis. The EFA returned a 4-factor solution which explained

Table 3 Comparisons of Arabic-COHIP-SF 19 and subscale scores by participants, caries status and oral health satisfaction, rating and perceived treatment need $(n=876)$

\begin{tabular}{|c|c|c|c|c|}
\hline & \multicolumn{4}{|c|}{ A-COHIP-SF 19 Overall scale, subscales } \\
\hline & Overall (19) & Oral health well-being & Functional well-being & Socio-emotional well-being \\
\hline Clinically assessed dental caries & Mean (SD) & & & \\
\hline Caries free $(n=406)$ & $62.13(12.42)$ & $15.37(4.30)$ & $14.51(3.03)$ & $32.25(8.19)$ \\
\hline Caries active $(n=355)$ & $60.28(13.38)$ & $14.36(4.76)$ & $14.13(3.04)$ & $31.78(8.67)$ \\
\hline$P$ value & $0.033^{*}$ & $0.002^{* *}$ & $0.031^{*}$ & 0.569 \\
\hline Participants' satisfaction with oral health & Mean (SD) & & & \\
\hline Satisfied $(n=665)$ & $62.91(11.56)$ & $15.09(4.43)$ & $14.63(2.67)$ & $33.17(7.57)$ \\
\hline Not satisfied $(n=211)$ & $55.56(15.41)$ & $13.98(4.94)$ & $13.27(3.85)$ & $28.31(9.94)$ \\
\hline$P$ value & $\leq 0.001^{* * *}$ & $0.005^{* *}$ & $\leq 0.001^{* * *}$ & $\leq 0.001^{* * *}$ \\
\hline \multicolumn{5}{|l|}{$\begin{array}{l}\text { Participants' perceived oral health } \\
\text { treatment needs }\end{array}$} \\
\hline Yes (393) & $56.81(14.54)$ & $13.41(4.80)$ & $13.58(3.61)$ & $29.81(9.66)$ \\
\hline No (483) & $64.65(10.29)$ & $15.81(4.05)$ & $14.89(2.35)$ & $33.78(6.83)$ \\
\hline$P$ value & $\leq 0.001^{* * *}$ & $\leq 0.001^{* * *}$ & $\leq 0.001^{* * *}$ & $\leq 0.001^{* * *}$ \\
\hline \multicolumn{5}{|l|}{ Self-rated oral health } \\
\hline Excellent/good $(n=584)$ & $64.51(9.84)$ & $15.61(4.03)$ & $14.78(2.43)$ & $34.12(6.54)$ \\
\hline Poor $(n=292)$ & $54.39(15.60)$ & $13.35(5.18)$ & $13.35(3.73)$ & $27.76(10.10)$ \\
\hline$P$ value & $\leq 0.001^{* * *}$ & $\leq 0.001^{* * *}$ & $\leq 0.001^{* * *}$ & $\leq 0.001^{* * *}$ \\
\hline
\end{tabular}

Man-Whitney $\mathrm{U}$ test was used to compare the subgroups, ${ }^{*} P \leq 0.05,{ }^{* *} P \leq 0.01,{ }^{* * *} P \leq 0.001$ 
Table 4 Mean scores, item impacts and item-total correlations and EFA findings. Responses to how often have you experienced the following in last 3 months? $(n=876)$

\begin{tabular}{|c|c|c|c|c|c|c|c|c|c|}
\hline \multirow{2}{*}{$\begin{array}{l}\text { Item } \\
\text { Oral Health—Well-being }\end{array}$} & \multirow[t]{2}{*}{ Mean } & \multirow[t]{2}{*}{ SD } & \multirow[t]{2}{*}{$\begin{array}{l}\% \text { of participants } \\
\text { reported the impact }\end{array}$} & \multirow[t]{2}{*}{ Item impact } & \multirow[t]{2}{*}{$\begin{array}{l}\text { Item total } \\
\text { correlation }\end{array}$} & \multicolumn{4}{|c|}{ EFA component } \\
\hline & & & & & & 1 & 2 & 3 & 4 \\
\hline Had pain in your teeth/toothache. & 1.3 & 1.4 & 49.9 & 64.9 & 0.44 & & 0.51 & & \\
\hline discoloured teeth & 0.8 & 1.3 & 24.5 & 19.6 & 0.36 & & & 0.62 & \\
\hline crooked teeth & 1.1 & 1.6 & 33.1 & 36.4 & 0.39 & & & 0.59 & \\
\hline Had bad breath. & 0.8 & 1.2 & 28.1 & 22.5 & 0.43 & & & 0.58 & \\
\hline Bleeding gums. & 1.22 & 1.5 & 43.0 & 52.6 & 0.33 & & & & \\
\hline \multicolumn{10}{|l|}{ Functional Well-being } \\
\hline $\begin{array}{l}\text { Had difficulty eating foods you } \\
\text { would like to eat }\end{array}$ & 0.7 & 1.3 & 23.5 & 16.5 & 0.48 & & 0.69 & & \\
\hline trouble sleeping & 0.3 & 0.9 & 12.6 & 3.4 & 0.49 & & 0.67 & & \\
\hline difficultly saying certain words & 0.3 & 0.9 & 8.6 & 2.6 & 0.36 & & 0.61 & & \\
\hline difficulty keeping your teeth clean & 0.4 & 1.1 & 13.8 & 5.52 & 0.41 & & 0.61 & & \\
\hline \multicolumn{10}{|l|}{ Socio-emotional Well-being } \\
\hline unhappy or sad & 0.7 & 1.4 & 22.4 & 15.7 & 0.65 & 0.74 & & & \\
\hline Felt worried or anxious & 0.7 & 1.6 & 21.1 & 14.8 & 0.72 & 0.78 & & & \\
\hline Avoided smiling or laughing & 0.6 & 1.3 & 18.0 & 10.8 & 0.69 & 0.80 & & & \\
\hline Felt that you look different & 0.5 & 1.2 & 15.2 & 7.6 & 0.69 & 0.84 & & & \\
\hline worried about what other people think & 0.5 & 1.2 & 15.1 & 7.6 & 0.64 & 0.82 & & & \\
\hline Been teased or bullied by peers & 0.5 & 1.2 & 14.8 & 7.4 & 0.64 & 0.79 & & & \\
\hline Missed School for any reason & 0.2 & 0.7 & 6.8 & 1.4 & 0.34 & 0.61 & & & \\
\hline $\begin{array}{l}\text { Not wanted to speak/read out } \\
\text { loud in class }\end{array}$ & 0.3 & 1.0 & 9.9 & 3.0 & 0.51 & 0.53 & & & \\
\hline Been confident & 2.0 & 1.9 & 54.1 & 108.2 & 0.19 & & & & 0.92 \\
\hline Felt attractive & 2.0 & 1.8 & 56.2 & 112.4 & 0.20 & & & & 0.91 \\
\hline
\end{tabular}

$57 \%$ of data diversity. The item "bleeding gum" was eliminated. The item "pain" was grouped with Functional items. The change from the original 3-factor COHIP-19 was the addition of a new sub-scale which comprised of the items related to "Been confident" and "Felt that you were attractive". Interestingly, as well as forming a separate sub-scale, these two items also showed the highest factor loadings, highest item impact scores and least total item correlation values (0.19 and 0.20 , respectively). High item impacts were also observed for the dental pain and gingival bleeding Items.

\section{Discussion}

The purpose of this study was to cross-culturally adapt the original English-language COHIP-SF19 to an Arabic cultural context and to test the psychometric properties of Arabic COHIP-SF19 in a population-based sample of Libyan schoolchildren. In reviewing the literature, only one study, conducted in China, has touched on testing COHIP-SF19 performance in a different culture [24]. To the authors' knowledge, the present study is the first in an Arabic speaking country. The Arabic COHIP-SF19 was successfully developed and cross-culturally adapted, showing satisfactory equivalence and psychometric properties in comparison to the original English version.

The Arabic COHIP-SF19 demonstrated excellent 'semantic equivalence' to the original English version. Although it is not uncommon to face translation difficulties when cross-culturally adapt OHRQoL questionnaires from English to the Arabic language [34], the translation process in the current study was trouble-free. This observation can be traced back to the development of original COHIP-SF19 where items with content overlap were identified and eliminated [22]. The review committee was satisfied with the wording and the vocabulary used in the Arabic COHIP-SF19, which indicates excellent content and face validity.

The Arabic COHIP-SF19 showed satisfactory 'item,'conceptual' and 'operational' equivalence. The participants in the pre-testing pilot reported that the questionnaire was clear, easy to use and relevant to its purpose. There was no need to modify the questionnaire's instructions, mode of administration or response options. It is worth noting, however, that the study participants were all similar in 
education level and taught in Arabic language up to the Sixth-grade level. It is therefore possible that these findings may not apply to someone with limited literacy skills who may require assisted or interview mode of administration rather than self-completion [35].

The Arabic COHIP-SF 19 exhibited acceptable level of internal consistency as measured by Cronbach's (0.85) which is comparable to that reported for the original English COHIP-SF19 ${ }^{22}$ and for the Chinese version [24]. At the subscales level, only the Socioemotional wellbeing scale showed acceptable value of Cronbach's alpha (0.83). The Cronbach's alpha values for Oral health and Functional well-being subscales were quite lower, although they were higher than those observed in the Chinese study [24]. However, items interrelatedness in these two subscales was acceptable (above the recommended level of 0.2 [36]). Therefore, low Cronbach's alpha values, observed in the current study, may have something to do with the small numbers of items in Oral health and Functional well-being subscales [37]. The test-retest reliability for the overall scale of Arabic COHIP-SF19 was substantial, above the recommended threshold [38], indicating very good reproducibility for the Arabic COHIP-SF19 [19]. The ICC score for the overall scale was 0.76 which is and comparable to that found in the Chinese study [24] .

Construct validity was examined by testing the associations between Arabic COHIP-SF19 and clinical caries data and global ratings of oral health. Almost all predefined hypotheses were confirmed. The Arabic COHIP-SF19 was able to distinguish between subgroups according to their caries status. Our data show that caries active participants appeared to have lower COHIP-SF19 scores than their caries-free peers. In the current study, lower scores of COHIP-SF 19 were observed among those who rated oral health as 'poor/ very poor', felt unsatisfied with their oral health and who perceived the need of dental treatment. These findings are in keeping with previous studies of COHIP-SF19 [22, 24], and suggest satisfactory construct validity.

EFA indicated that the Arabic version is characterized by 4 dimensions instead of the 3 dimensions suggested in the development study of original COHIP-SF19. The new dimension comprised 2 items related to self-image, which also showed high loading and item impact than other items in the scale. Current data does not allow for a plausible explanation to this finding but it may have something to do with variations in the characteristics of the study population [39]. The current study sample was recruited from community setting wherein oral health and function issues may not be as high as if the sample was recruited from a clinical setting. Unfortunately, previous studies of COHIP-SF19 did not report on item impact and factorial validity which precluded the comparison with our findings. Further research, however, is required to compare the 4- and 3 -factor CHOHP-SF in community and clinical based samples.

The average score for Arabic COHIP-SF19 was relatively high $(61.13 \pm 12.97)$. This score is higher than that observed in the original study and among the Chinese children [22, 24], and suggests low oral health impacts among Libyan school children, which is not uncommon for children from Arabic speaking countries [40, 41]. In the current study, females were more likely to experience oral health impacts than their male counterparts. A similar trend has been observed in the dental literature on OHRQoL among children [34, 41-43]. Although it is well recognized in the general literature that females are more sensitive than males because of several biological, cultural, psychological, and social factors [44], gender differences in perception of OHRQoL should be taken in account when developing oral health interventions and programs.

The overall scale of Arabic COHIP-SF19 demonstrated a lack of floor and ceiling effects which reflects the validity and reliability of the response scale [31]. Interestingly, the ceiling effect existed in the subscales, which was frequently achieved in the Function well-being subscale. It is difficult to explain this observation, but it may have something to do with how the participants define what constitutes an optimum oral health. It is well recognized that individual's appraisal of the quality of life is influenced by the extent to which expectations and goals are matched by experience [45]. The current study was conducted in a conflict-affected country which colors all aspects of live and hence the perception of oral health importance and impacts. Therefore, it could be the case that the participants gave higher ratings to functional impacts than they give to social and emotional impacts of oral health [40]. However, more qualitative work is required to further explore this phenomenon.

As for all cross-sectional studies, this study has some inherent limitations, specifically related to the evaluative performance of the Arabic COHIP-SF19. For example, it was impossible to assess the responsiveness of the Arabic COHIP-SF19, which has important implications for studies using OHRQoL as an evaluative outcome measure such as interventional studies and longitudinal observational studies aiming to improve oral health care [3]. In addition, the participants were limited to the 12year-old age group, and hence age-related variations were not explored. Therefore, using longitudinal research design and including various age groups should be considered in future research. 


\section{Conclusion}

Using a comprehensive cross-cultural adaptation process, the original English language COHIP-SF 19 was successfully translated and adapted to the Arabic context. The Arabic COHIP-SF 19 is satisfactorily equivalent to the original version and is valid and reliable to estimate OHRQoL in Arabic schoolchildren. The Arabic COHIP-SF 19, therefore, can be used to assess subjective oral health needs among Libyan children as part of national surveys and clinical assessment in dental practice. However, the EFA suggested some modifications to the subscales which has been identified as an area of further assessment. Further research is required to investigate the longitudinal validity and responsiveness of Arabic COHIP-SF 19 as well as its performance among children from different age groups.

\section{Acknowledgements}

The authors wish to thank all the children and their parents for participating in the study and the translators who helped in the development of the Arabic version of COHIP-SF 19. We also thank Dr. Ala Aldarat, the dean of dental school at the University of Benghazi for his generous help in conducting this study.

\section{Funding}

The authors declare that have not received any funding in relation to this study.

\section{Availability of data and materials}

The datasets used and/or analysed during the current study are available from the corresponding author on reasonable request.

\section{Authors' contributions}

AA: Study design, conception and data analysis and interpretation and manuscript writing and revision. SB: Data analysis and interpretation and manuscript writing and critically revised the article. LB: Development and piloting of Arabic questionnaire, data collection and interpretation. SF: Development and piloting of Arabic questionnaire data collection and interpretation. IA: study design, data collection and interpretation. RH: Data analysis and interpretation and manuscript writing and critically revised the article. All authors read and approved the final manuscript.

\section{Ethics approval and consent to participate}

Ethical clearance and permissions for the study were obtained from Ethics committee at the university of Liverpool and Faculty of Dentistry at the University of Benghazi prior to data collection. Informed consent was obtained from all individual participants included in the study.

\section{Consent for publication}

Not applicable

\section{Competing interests}

The authors declare that they have no competing interests.

\section{Publisher's Note}

Springer Nature remains neutral with regard to jurisdictional claims in published maps and institutional affiliations.

\section{Author details}

'Department of Health Services Research University of Liverpool, Liverpool, UK. ${ }^{2}$ Unit of Dental Public Health, School of Dentistry, University of Sheffield, Sheffield, UK. ${ }^{3}$ Department of Community and Preventive Dentistry, Faculty of Dentistry, University of Benghazi, Benghazi, Libya.
Received: 12 July 2017 Accepted: 2 November 2017

Published online: 13 November 2017

\section{References}

1. Preedy VR, Watson RR. Handbook of disease burdens and quality of life measures. New York, NY: Springer New York; 2010

2. Glick M, Williams DM, Kleinman DV, Vujicic M, Watt RG, Weyant RJ. A new definition for oral health developed by the FDI world dental federation opens the door to a universal definition of oral health. Br Dent J. 2016; 221(12):792-3.

3. Sischo L, Broder HL. Oral health-related quality of life: what, why, how, and future implications. J Dent Res. 2011;90(11):1264-70.

4. Sheiham A, Tsakos G: Oral health needs assessment. In., edn.: Quintessence; 2007.

5. Robinson PG. Choosing a measure of health related quality of life. Community Dent Health. 2016;33(2):107-15.

6. McGrath C, Bedi R. Understanding the value of oral health to people in Britain-importance to life quality. Community Dent Health. 2002;19(4):211-4.

7. Locker D, Frosina C, Murray H, Wiebe D, Wiebe P. Identifying children with dental care needs: evaluation of a targeted school-based dental screening program. J Public Health Dent. 2004;64(2):63-70

8. Jokovic A, Locker D, Tompson B, Guyatt G. Questionnaire for measuring oral health-related quality of life in eight- to ten-year-old children. Pediatr Dent. 2004;26(6):512-8.

9. Locker D. Applications of self-reported assessments of oral health outcomes. J Dent Educ. 1996:60(6):494-500.

10. Masood M, Masood Y, Saub R, Newton JT. Need of minimal important difference for oral health-related quality of life measures. J Public Health Dent. 2014;74(1):13-20.

11. Gherunpong S, Tsakos G, Sheiham A. A sociodental approach to assessing dental needs of children: concept and models. Int J Paediatr Dent. 2006; 16(2):81-8.

12. Petersen PE, Bourgeois D, Ogawa H, Estupinan-Day S, Ndiaye C. The global burden of oral diseases and risks to oral health. Bull World Health Organ. 2005:83(9):661-9.

13. Marcenes W, Kassebaum NJ, Bernabe E, Flaxman A, Naghavi M, Lopez A, Murray CJ. Global burden of oral conditions in 1990-2010: a systematic analysis. J Dent Res. 2013;92(7):592-7.

14. Selwitz RH, Ismail Al, Pitts NB. Dental caries. Lancet. 2007:369(9555):51-9.

15. Tickle M, Blinkhorn AS, Milsom KM. The occurrence of dental pain and extractions over a 3-year period in a cohort of children aged 3-6 years. J Public Health Dent. 2008;68(2):63-9.

16. Pine CM, Harris RV, Burnside G, Merrett MCW. An investigation of the relationship between untreated decayed teeth and dental sepsis in 5-yearold children. Br Dent J. 2006;200(1):45-7.

17. Jackson SL, Vann WF Jr, Kotch JB, Pahel BT, Lee JY. Impact of poor oral health on children's school attendance and performance. Am J Public Health. 2011;101(10):1900-6.

18. Goodwin M, Sanders C, Davies G, Walsh T, Pretty IA. Issues arising following a referral and subsequent wait for extraction under general anaesthetic: impact on children. BMC Oral Health. 2015;15(1):3.

19. Gilchrist F, Rodd H, Deery C, Marshman Z. Assessment of the quality of measures of child oral health-related quality of life. BMC Oral Health. 2014; 14(1):40.

20. Jokovic A, Locker D, Stephens M, Kenny D, Tompson B, Guyatt G. Validity and reliability of a questionnaire for measuring child oral-health-related quality of life. J Dent Res. 2002;81(7):459-63.

21. Broder HL, McGrath C, Cisneros GJ. Questionnaire development: face validity and item impact testing of the Child Oral Health Impact Profile. Community Dent Oral Epidemiol. 2007;35(Suppl 1):8-19.

22. Broder HL, Wilson-Genderson M, Sischo L. Reliability and validity testing for the child oral health impact profile-reduced (COHIP-SF 19). J Public Health Dent. 2012;72(4):302-12.

23. Jokovic A, Locker D, Guyatt G. Short forms of the child perceptions questionnaire for 11-14-year-old children (CPQ(11-14)): development and initial evaluation. Health Qual Life Outcomes. 2006;4(1):4.

24. Li C, Xia B, Wang Y, Guan X, Yuan J, Ge L. Translation and psychometric properties of the Chinese (mandarin) version of the child oral health impact profile-short form 19 (COHIP-SF 19) for school-age children. Health Qual Life Outcomes. 2014;12(1):169. 
25. Beaton DE, Bombardier C, Guillemin F, Ferraz MB. Guidelines for the process of cross-cultural adaptation of self-report measures. Spine. 2000;25(24):3186-91.

26. Herdman M, Fox-Rushby J, Badia X. A model of equivalence in the cultural adaptation of HRQoL instruments: the universalist approach. Qual Life Res. 1998; $7(4): 323-35$.

27. Guillemin F, Bombardier C, Beaton D. Cross-cultural adaptation of healthrelated quality of life measures: literature review and proposed guidelines. J Clin Epidemiol. 1993;46(12):1417-32.

28. Charter RA. Sample size requirements for precise estimates of reliability, generalizability, and validity coefficients. J Clin Exp Neuropsychol. 1999;21(4): $559-66$

29. Ismail Al, Sohn W, Tellez M, Amaya A, Sen A, Hasson H, Pitts NB. The international caries detection and assessment system (ICDAS): an integrated system for measuring dental caries. Community Dent Oral Epidemiol. 2007; 35(3):170-8.

30. Locker D, Slade G. Oral health and the quality of life among older adults: the oral health impact profile. J Can Dent Assoc. 1993;59(10):830-3. 837838,844

31. Terwee $C B$, Bot $S D$, de Boer MR, van der Windt DA, Knol DL, Dekker J, Bouter LM, de Vet HC. Quality criteria were proposed for measurement properties of health status questionnaires. J Clin Epidemiol. 2007;60(1):34-42.

32. Osborne JW, Costello AB. Best practices in exploratory factor analysis: four recommendations for getting the most from your analysis. Pract Assess, Res Eval. 2005;10:7.

33. Boiko OV, Baker SR, Gibson BJ, Locker D, Sufi F, Barlow AP, Robinson PG. Construction and validation of the quality of life measure for dentine hypersensitivity (DHEQ). J Clin Periodontol. 2010;37(11):973-80.

34. Farsi NJ, El-Housseiny AA, Farsi DJ, Farsi NM. Validation of the Arabic version of the early childhood oral health impact scale (ECOHIS). BMC Oral Health. 2017;17(1):60.

35. Saub R, Locker D, Allison P, Disman M. Cross-cultural adaptation of the oral health impact profile (OHIP) for the Malaysian adult population. Community Dent Health. 2007;24(3):166-75.

36. Kline P. Handbook of psychological testing. London: Routledge; 2013.

37. Streiner DL. Starting at the beginning: an introduction to coefficient alpha and internal consistency. J Pers Assess. 2003;80(1):99-103.

38. Nunnally JC. The assessment of reliability. In: Nunnally JC, Bernstein I, editors. Psychometric theory. 3rd ed. New York: McGraw-Hill; 1994. p. 248-92.

39. John MT, Reissmann DR, Feuerstahler L, Waller N, Baba K, Larsson P, Celebic A, Szabo G, Rener-Sitar K. Exploratory factor analysis of the oral health impact profile. J Oral Rehabil. 2014;41(9):635-43.

40. Brown A, Al-Khayal Z. Validity and reliability of the Arabic translation of the child oral-health-related quality of life questionnaire (CPQ11-14) in Saudi Arabia. Int J Paediatr Dent. 2006;16(6):405-11.

41. Nurelhuda NM, Ahmed MF, Trovik TA, Astrom AN. Evaluation of oral healthrelated quality of life among Sudanese schoolchildren using child-OIDP inventory. Health Qual Life Outcomes. 2010;8(1):152.

42. de Oliveira CM, Sheiham A: Orthodontic treatment and its impact on oral health-related quality of life in Brazilian adolescents. J Orthod 2004, 31(1): 20-27; discussion 15.

43. de Souza Barbosa T, Gaviao MB, Castelo PM, Leme MS. Factors associated with oral health-related quality of life in children and preadolescents: a cross-sectional study. Oral Health Prev Dent. 2016;14(2):137-48.

44. Riley JLRM, Wise EA, Myers CD, Fillingim RB. Sex differences in the perception of noxious experimental stimuli: a meta-analysis. Pain. 1998; 74(2-3):81-7.

45. Albrecht GL, Devlieger PJ. The disability paradox: high quality of life against all odds. Soc Sci Med. 1999;48(8):977-88.

\section{Submit your next manuscript to BioMed Central and we will help you at every step:}

- We accept pre-submission inquiries

- Our selector tool helps you to find the most relevant journal

- We provide round the clock customer support

- Convenient online submission

- Thorough peer review

- Inclusion in PubMed and all major indexing services

- Maximum visibility for your research

Submit your manuscript at www.biomedcentral.com/submit

Biomed Central 